\title{
Hyperglycaemia on admission to hospital and COVID-19
}

\section{Celestino Sardu ${ }^{1}$ - Nunzia D'Onofrio ${ }^{2}$ - Maria Luisa Balestrieri ${ }^{2} \cdot$ Michelangela Barbieri $^{1}$ - Maria Rosaria Rizzo ${ }^{1}$. Vincenzo Messina ${ }^{3} \cdot$ Paolo Maggi $^{3} \cdot$ Nicola Coppola $^{4} \cdot$ Giuseppe Paolisso $^{1} \cdot$ Raffaele Marfella $^{1}$ (D)}

Received: 12 June 2020 / Accepted: 18 June 2020 / Published online: 6 July 2020

(C) The Author(s) 2020

Keywords Covid-19 $\cdot$ Hyperglycaemia $\cdot$ Inflammatory cytokines $\cdot$ Mechanical ventilation $\cdot$ Non-invasive ventilation

\author{
Abbreviation \\ COVID-19 Coronavirus disease-2019
}

To the Editor: We read with great interest the article by Bertrand Cariou and coworkers on people with diabetes hospitalised for coronavirus disease-2019 (COVID-19) [1]. The analysis of the data obtained on a very large population (1317 patients) provided important information, highlighting a role for BMI in disease severity, but did not correctly interpret the data on the role of hyperglycaemia at admission to hospital. In fact, the authors observed an age- and sex-independent association between increased admission plasma glucose and the severity of COVID-19. Nevertheless, they postulate that this observation is rather the consequence of the severity of the infection than a causal primary factor'. This interpretation of the data underestimates the importance of glycaemic control in COVID-19 and may result in caregivers focusing attention on therapeutic management of hyperglycaemic patients. To investigate the importance of glucose management of COVID-19 patients, we analysed recent data on glycaemic control and COVID-19 outcomes. Acute hyperglycaemia occurs in about $50 \%$ of patients hospitalised for COVID-19, while the prevalence of diabetes is about $7 \%$ [2]. Moreover, increased plasma glucose on admission to hospital was associated with poorer outcomes in

Raffaele Marfella

raffaele.marfella@unicampania.it

1 Department of Advanced Medical and Surgical Sciences (DAMSS), University of Campania 'Luigi Vanvitelli', 80138 Naples, Italy

2 Department of Precision Medicine, University of Campania 'Luigi Vanvitelli', Naples, Italy

3 Department of Infectious Diseases, Sant'Anna Hospital, Caserta, Italy

4 Department of Mental and Physical Health and Preventive Medicine, University of Campania 'Luigi Vanvitelli', Naples, Italy patients with mild, moderate and severe COVID-19 [2-4]. Increased concentrations of proinflammatory cytokines, including IL-2 receptors (IL-2Rs), IL-6 and TNF- $\alpha$, may have a pivotal role in the mechanism(s) underlying this association. Elevated levels of these proteins were common in both COVID-19 and in hyperglycaemia [5-7]. Thus, an over-activation of the innate immune system may be responsible for poorer outcomes in hyperglycaemic patients affected by COVID-19, including those treated with tocilizumab, an inhibitor of IL-6 [7]. Therefore, to date, despite full therapy, including oxygen therapy, hydroxychloroquine and antiviral treatment, hyperglycaemic patients with COVID-19 have a worse prognosis. In this context, we hypothesise that achievement of rapid control of hyperglycaemia in the first $24 \mathrm{~h}$ may improve outcomes. To investigate the role of early glycaemic control in the outcomes of patients with COVID-19, we studied 132 consecutive hospitalised hyperglycaemic patients with moderate disease, admitted to Infectious diseases departments (Vanvitelli University, Naples Italy; San Sebastiano Caserta Hospital, Caserta, Italy). Patients were grouped as being with or without severe disease according to post hoc analysis. Hyperglycaemia was defined as plasma glucose level on admission of $>7.7 \mathrm{mmol} / \mathrm{l}$. Severity of COVID-19 infection was categorised as follows: mild, patients with fever and no evidence of pneumonia on imaging; moderate, patients with fever, respiratory tract symptoms, pneumonia on imaging without the need for invasive ventilation; critical, occurrence of respiratory failure requiring mechanical ventilation, presence of shock, other organ failure requiring monitoring and treatment in the intensive care unit. Severe disease as a composite endpoint was admission to an intensive care unit, the need for mechanical ventilation or death. Data are presented as mean $\pm \mathrm{SD}$, categorical variables were summarised as counts and percentages. According to the post hoc analysis, patients were grouped as being without severe disease $(n=72)$ or with severe disease $(n=60)$. The study protocol was approved by the ethics committee of the University of Campania 'Luigi Vanvitelli'. Written informed consent was 
Fig. 1 (a) Blood glucose levels on admission to hospital and after $24 \mathrm{~h}$ for patients with $(n=60)$ and without severe disease $(n=72)$ at 20 days after hospitalisation. (b) Blood glucose changes between baseline and $24 \mathrm{~h}$ for patients with and without severe disease at 20 days after hospitalisation. Boxand-whisker plots display the median, 25th and 75th percentiles and range. $* p<0.05$ by Student's $t$ test; all calculations were performed using SPSS version 23 software (IBM, Armonk, NY, USA) a

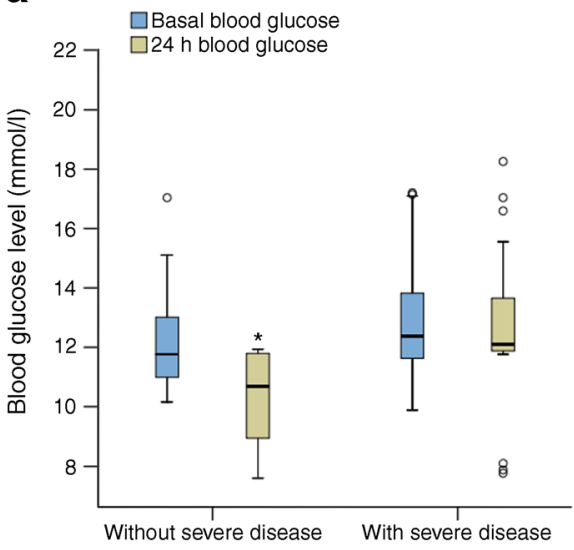

b

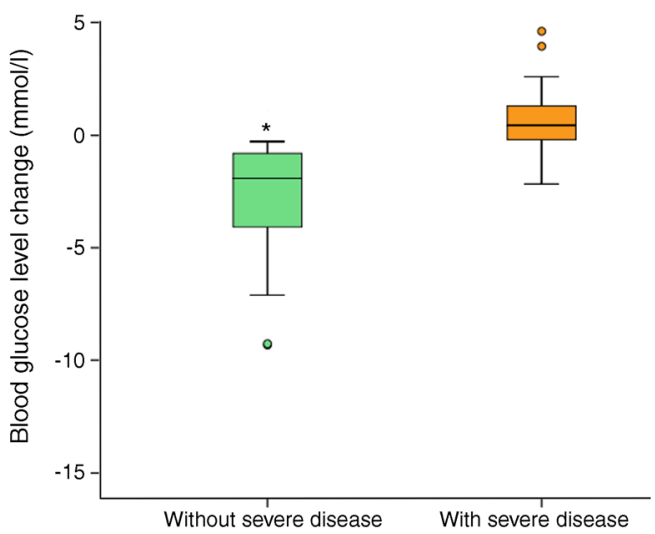

obtained from all patients. There were no significant differences in clinical characteristics between the groups with and without severe disease (age: $64.9 \pm 7.1$ vs $65.8 \pm 6.2$ years, $p=0.997$; BMI: $28.6 \pm 1.9$ vs $28.3 \pm 1.9 \mathrm{~kg} / \mathrm{m}^{2}, p=0.877$; men: $79.2 \%$ vs $84.2 \%, p=0.263$; previous diabetes diagnosis, $19.4 \%$ vs $25.0 \%$, $p=0.288$; insulin treatment on admission in patients with previous diabetes diagnosis without severe disease $n=14 / 50$ [28\%] vs with severe disease $n=15 / 45$ [33.3\%], $p=0.102$; oral glucoselowering drugs $n=43$ [86\%] vs $n=42$ [93.3\%], $p=0.096$ ). When analysing blood glucose levels on admission and after $24 \mathrm{~h}$, we found that a decrease in glucose levels between baseline and $24 \mathrm{~h}$ was associated with a lower rate of progression to severe disease and death at 20 days in both nondiabetic and diabetic hyperglycaemic patients (Fig. 1). Thus, to date, early glycaemic control may be a suitable therapeutic option to reduce the poor outcomes in hospitalised hyperglycaemic COVID-19 patients with or without a previous diabetes diagnosis [8].

Acknowledgements We are grateful to L. Ruggiero (Department of Advanced Medical and Surgical Sciences (DAMSS), University of Campania 'Luigi Vanvitelli')) for his important work in the clinical evaluation of patients admitted to our department. We are also grateful to F. Miglietta (Chair of Geriatrics, University of Campania 'Luigi Vanvitelli') for critical analysis of the clinical data.

Data availability The data are available on request from the authors.

Funding This research received no specific grant from any funding agency in the public, commercial or not-for-profit sectors.

Authors' relationships and activities The authors declare that there are no relationships or activities that might bias, or be perceived to bias, their work.

Contribution statement All authors were responsible for drafting the article and revising it critically for important intellectual content. All authors approved the version to be published. RM takes full responsibility for the work as a whole, including the study design, access to data and the decision to submit and publish the manuscript.

Open Access This article is licensed under a Creative Commons Attribution 4.0 International License, which permits use, sharing, adaptation, distribution and reproduction in any medium or format, as long as you give appropriate credit to the original author(s) and the source, provide a link to the Creative Commons licence, and indicate if changes were made. The images or other third party material in this article are included in the article's Creative Commons licence, unless indicated otherwise in a credit line to the material. If material is not included in the article's Creative Commons licence and your intended use is not permitted by statutory regulation or exceeds the permitted use, you will need to obtain permission directly from the copyright holder. To view a copy of this licence, visit http://creativecommons.org/licenses/by/4.0/.

\section{References}

1. Cariou B, Hadjadj S, Wargny M et al (2020) Phenotypic characteristics and prognosis of inpatients with COVID-19 and diabetes: the CORONADO study. Diabetologia. https://doi.org/10.1007/s00125020-05180-X

2. Li X, Xu S, Yu M et al (2020) Risk factors for severity and mortality in adult COVID-19 inpatients in Wuhan. J Allergy Clin Immunol. https://doi.org/10.1016/j.jaci.2020.04.006

3. Zhu L, She Z, Cheng X et al (2020) Association of blood glucose control and outcomes in patients with COVID-19 and pre-existing type 2 diabetes. Cell Metab 31(6):1068-1077.e3. https://doi.org/10. 1016/j.cmet.2020.04.021

4. Sardu C, D’Onofrio N, Balestrieri ML et al (2020) Outcomes in hyperglycemic patients affected by Covid-19: can we do more on glycemia control? Diabetes Care 43. https://doi.org/10.2337/DC20-0723

5. Zhang C, Wu Z, Li J-W, Zhao H, Wang G-Q (2020) The cytokine release syndrome (CRS) of severe COVID-19 and interleukin-6 receptor (IL-6R) antagonist tocilizumab may be the key to reduce the mortality. Int $\mathrm{J}$ Antimicrob Agents. https://doi.org/10.1016/j.ijantimicag.2020.105954

6. Bingwen L, Min L, Zhiguang Z, Xuan G, Yufei X (2020) Can we use interleukin-6 (IL-6) blockade for coronavirus disease 2019 (COVID19)-induced cytokine release syndrome (CRS)? J Autoimmun. https://doi.org/10.1016/j.jaut.2020.102452

7. Marfella R, Paolisso P, Sardu C et al (2020) Negative impact of hyperglycaemia on tocilizumab therapy in Covid-19 patients. Diabetes Metab. https://doi.org/10.1016/j.diabet.2020.05.005

8. Shenav-Zaltzman G, Segal G, Konvalina N, Tirosh A (2020) Remote glucose monitoring of hospitalized, quarantined patients with diabetes and COVID-19. Diabetes Care 43. https://doi.org/10.2337/DC20-0696

Publisher's note Springer Nature remains neutral with regard to jurisdictional claims in published maps and institutional affiliations. 